\title{
Convergence of Finite Difference Scheme and Analytic Data
}

\author{
By \\ Kantaro HAYAKAWA*
}

\section{§ 0. Introduction}

Finite difference methods are used widely to approximate solutions of partial differential equations numerically. To build up the difference scheme from the partial differential equation, we should be careful. Formal correspondence of difference schemes to the differential equation is not sufficient for the convergence of the solutions to the exact one. For equations of hyperbolic type, the convergence is assured under the CFL condition [1], which is concerned with the ratio of the space increment to the time one.

However, this does not assert that, without CFL condition, every discrete solution diverges. G. Dahlquist [2] exposed an interesting example. He considered the wave equation, and descretized it by the usual finite difference scheme. He showed that if initial data for the wave equation are real analytic, then solutions of difference scheme converge to the solution of the wave equation, even in the absence of the CFL condition.

Our results in this issue is in the same direction. Our aim is to show that, in the analytic case, we need not the CFL type condition for the convergence of solutions of the finite difference scheme.

We treat of the linear first order system with constant coefficients;

$$
\left\{\begin{array}{l}
\frac{\partial}{\partial x_{1}} U=A \frac{\partial}{\partial x_{2}} U \\
U\left(0, x_{2}\right)=U_{0}\left(x_{2}\right)
\end{array}\right.
$$

where $U=U\left(x_{1}, x_{2}\right)$ is an unknown $m$-vector valued function, $A$ a given $m \times m$ constant real matrix, $U_{0}\left(x_{2}\right)$ a given $m$-vector valuted function. The discretization by the simple finite difference method of $(0.1)$ is

$$
\left\{\begin{array}{l}
\frac{1}{\Delta x_{1}}\left\{\tilde{U}\left(x_{1}+\Delta x_{1}, x_{2}\right)-\tilde{U}\left(x_{1}, x_{2}\right)\right\}=\frac{1}{\Delta x_{2}} A\left\{\tilde{U}\left(x_{1}, x_{2}+\Delta x_{2}\right)-\tilde{U}\left(x_{1}, x_{2}\right)\right\} \\
\tilde{U}\left(0, x_{2}\right)=U_{0}\left(x_{2}\right)
\end{array}\right.
$$

Communicated by S. Hitotumatu, January 19, 1988.

* Department of Applied Mathematics, Faculty of Engineering Science, Osaka University, Osaka 560, Japan. 
Here, $\Delta x_{1}$ and $\Delta x_{2}$ are nonzero increments which are independent of $x_{1}, x_{2}$.

Our purpose is to show that, if $U_{0}\left(x_{2}\right)$ is analytic at $x_{2}=0$, then the solution $\tilde{U}\left(x_{1}, x_{2}\right)$ of $(0.2)$ converges to the solution of $(0.1)$ in the neighbourhood of $\left(x_{1}, x_{2}\right)=(0,0)$.

G. Dahlquist utilized the Fourier transform in [2]. We'll show our results rather through the direct way-the algebraic calculous of power series. It should be noticed that we made no assumptions on the constant real matrix A, especially no hyperbolicity. Thus, even when $A=\left(\begin{array}{rr}0 & 1 \\ -1 & 0\end{array}\right)$, i. e. Cauchy-Riemann equation, our theorem stands true. The CFL condition is concerned with the relation between the ratio $\Delta x_{1} / \Delta x_{2}$ and the hyperbolicity of $(0.1)$. So, this CFL condition has no meanings in our case.

In the next section, we give some notations, and precise discription of our results. In $\S 2$, we prove our results.

\section{$\S 1$. Notations and Results}

For $m \times m$ constant matrix $A=\left(a_{j, k}\right)_{j \downarrow, k \rightarrow}$ and $m$-vector $U=\left(u_{j}\right)_{j \downarrow}$, we define $\|A\|,\|U\|$ by

$$
\begin{aligned}
& \|A\|=\operatorname{Max}\left\{\sum_{k=1}^{m}\left|a_{j, k}\right| ; j=1,2, \cdots, m\right\}, \\
& \|U\|=\operatorname{Max}\left\{\left|u_{j}\right| ; \quad j=1,2, \cdots, m\right\} .
\end{aligned}
$$

For a positive $\bar{x}_{1}$ and positive integer $N$, we set

$$
h_{1}=\frac{\bar{x}_{1}}{N} \text {. }
$$

For some non zero real $\tau$, we set

$$
h_{2}=\frac{h_{1}}{\tau} .
$$

We denote by $\hat{U}_{j, k}$ the value of solution $\tilde{U}$ of $(0.2)$ at $\left(x_{1}, x_{2}\right)=\left(j h_{1}, k h_{2}\right)$. For any real $\xi,[\xi]$ is the maximum integer which is not larger than $\xi$. For any positive real $x_{1}$ and any real $x_{2}$, we set

$$
\begin{aligned}
& j\left(x_{1}\right)=\left[\frac{x_{1}}{h_{1}}\right], \\
& k\left(x_{2}\right)=\left[\frac{x_{2}}{h_{2}}\right] .
\end{aligned}
$$

Let the initial function $U_{0}\left(x_{2}\right)$ be given on $\left(-r_{0}, r_{0}\right)$. Then $\hat{U}_{j, k}$ satisfy, for integers $j, k$, 


$$
\begin{cases}\frac{1}{h_{1}}\left\{\hat{U}_{j+1, k}-\hat{U}_{j, k}\right\}=\frac{1}{h_{2}} A\left\{\hat{U}_{j, k+1}-\hat{U}_{j, k}\right\}, & 0 \leqq j \leqq\left[r_{0} /\left|h_{2}\right|\right]-|k|, \\ \hat{U}_{0, k}=U_{0}\left(k h_{2}\right) & |k| \leqq\left[r_{0} /\left|h_{2}\right|\right] .\end{cases}
$$

For a positive real $r_{1}$, we define the triangular domain

$$
D_{\tau, r_{1}}=\left\{\left(x_{1}, x_{2}\right) ; x_{1}>0,\left|x_{2}\right|+\frac{1+|\tau|\|A\|}{|\tau|} x_{1}<r_{1}\right\} \text {. }
$$

Now, our main result is

Theorem. Assume $U_{0}\left(x_{2}\right)$ is analytic at 0 and its radius of convergence of the Taylor expansion at 0 is $r_{0}$. Then, for any $r_{1}<r_{0}$, and $\left(x_{1}, x_{2}\right) \in D_{\tau}, r_{1}$, $\hat{U}_{j\left(x_{1}\right), k\left(x_{2}\right)}$ converges to the solution $\tilde{U}\left(x_{1}, x_{2}\right)$ of $(0.2)$ as $N$ tends to $\infty$. This convergence is uniform on $D_{\tau, r_{1}}$.

\section{$\S 2$. Proof of Theorem}

From (1.5), we have

$$
\left\{\begin{array}{l}
\hat{U}_{j+1, k}=(1-\tau A) \hat{U}_{j, k}+\tau A \hat{U}_{j, k+1} \\
\hat{U}_{0, k}=U_{0}\left(k h_{2}\right)
\end{array}\right.
$$

Thus, we have

$$
\hat{U}_{j, k}=\sum_{q=0}^{j}\left(\begin{array}{l}
j \\
q
\end{array}\right)(1-\tau A)^{j-q}(\tau A)^{q} \hat{U}_{0, k+q} \quad 0 \leqq j \leqq N,|k+j| \leqq\left[r_{0} /\left|h_{2}\right|\right] .
$$

Here $\left(\begin{array}{l}j \\ q\end{array}\right)$ means the binomial coefficient. First we prepare the algebraic lemma as follows. For a positive integer $j$ and a non-negative integer $l$, we set

$$
T_{j, l}(a, b)=\sum_{q=1}^{j}\left(\begin{array}{l}
j \\
q
\end{array}\right) q^{l} a^{j-q} b^{q} .
$$

Lemma. Let $j$ a positive integer and $l$ be a non-negative integer. Thus, we have

$$
T_{j, l}(a, b)=\sum_{r=1}^{\operatorname{Min}(j, l)} j(j-1) \cdots(j-r+1) C_{l, r}(a+b)^{\jmath-r} b^{r},
$$

where integer coefficients $C_{l, r}$ are independent of $j$ and satisfy

$$
1 \leqq C_{l, r} \leqq r^{l-r}\left(\begin{array}{c}
l-1 \\
r-1
\end{array}\right) \quad l \geqq r .
$$

Proof. Obviously we have following relations:

$$
\left\{\begin{array}{l}
T_{j, 0}(a, b)=(a+b)^{j}, \\
T_{j, l+1}(a, b)=b \frac{\partial}{\partial b} T_{j, l}(a, b)
\end{array}\right.
$$


From these we have (2.3), and $C_{l, r}$ satisfy

$$
\left\{\begin{array}{l}
C_{l+1, r}=C_{l, r-1}+r C_{l, r} \quad 2 \leqq r \leqq l, \\
C_{l+1,1}=C_{l, 1}=1 \\
C_{l+1, l+1}=C_{l, l}=1 .
\end{array}\right.
$$

We denote $r^{r-l} C_{l, r}$ by $d_{l, r}$, and we have

$$
d_{l+1, r}=\left(1-r^{-1}\right)^{l-r+1} d_{l, r-1}+d_{l, r}, \quad l \geqq r \geqq 1 .
$$

From this we get the following inequalities;

$$
d_{l, r}<d_{l+1, r}<d_{l, r}+d_{l, r-1}, \quad l \geqq r \geqq 1 \text { 。 }
$$

Through the induction, we have $1 \leqq d_{l, r} \leqq\left(\begin{array}{c}l-1 \\ r-1\end{array}\right)$. Estimate (2.4) can be derived from these.

Proposition. Let $U_{0}\left(x_{2}\right)=x_{2}^{l} U_{0, l}$, where $U_{0, l}$ is some constant m-vector. Then $\hat{U}_{j\left(x_{1}\right), k\left(x_{2}\right)} \rightarrow\left(x_{2}+x_{1} A\right)^{l} U_{0, l}$ as $N \rightarrow \infty$. The convergence is uniform on any bounded set in $R^{2}$.

Proof. From (2.2) we have

$$
\begin{aligned}
\hat{U}_{j\left(x_{1}\right), k\left(x_{2}\right)} & =\sum_{q=0}^{j\left(x_{1}\right)}\left(\begin{array}{l}
j\left(x_{1}\right) \\
q
\end{array}\right)(1-\tau A)^{j\left(x_{1}\right)-q}(\tau A)^{q}\left(k\left(x_{2}\right)+q\right)^{l} h_{2}^{l} U_{0, l} \\
& =\sum_{p=0}^{l}\left(\begin{array}{l}
l \\
p
\end{array}\right)\left\{\sum_{q=0}^{j\left(x_{1}\right)}\left(\begin{array}{l}
j\left(x_{1}\right) \\
q
\end{array}\right)(1-\tau A)^{j\left(x_{1}\right)-q}(\tau A)^{q} q^{l-p}\right\} k\left(x_{2}\right)^{p} h_{2}^{l} U_{0, l} .
\end{aligned}
$$

Using (2.3), this is equal to

(2.5) $\sum_{p=0}^{l} \sum_{r=1}^{\operatorname{Min}\left(j\left(x_{1}\right), l-p\right)}\left(\begin{array}{l}l \\ p\end{array}\right) j\left(x_{1}\right)\left(j\left(x_{1}\right)-1\right) \cdots\left(j\left(x_{1}\right)-r+1\right) C_{l-p, r}(\tau A)^{r} k\left(x_{2}\right)^{p} h_{2}^{l} U_{0, l}$.

Each term can be reformed as follows:

$$
\begin{aligned}
& \left(\begin{array}{l}
l \\
p
\end{array}\right) j\left(x_{1}\right)\left(j\left(x_{1}\right)-1\right) \cdots\left(j\left(x_{1}\right)-r+1\right) C_{l-p, r} k\left(x_{2}\right)^{p} h_{2}^{l}(\tau A)^{r} U_{0, l} \\
= & \left(\begin{array}{l}
l \\
p
\end{array}\right) j\left(x_{1}\right)\left(j\left(x_{1}\right)-1\right) \cdots\left(j\left(x_{1}\right)-r+1\right)\left(\frac{x_{1}}{h_{1}}\right)^{-r} k\left(x_{2}\right)^{p}\left(\frac{x_{2}}{h_{2}}\right)^{-p} h_{2}^{l-p-r} \\
& \times C_{l-p, r} x_{2}^{p}\left(x_{1} A\right)^{r} U_{0, l} .
\end{aligned}
$$

When we tend $N$ to $\infty$, we have

$$
j\left(x_{1}\right) \rightarrow \infty, \quad h_{2} \rightarrow 0, \quad j\left(x_{1}\right)\left(\frac{x_{1}}{h_{1}}\right)^{-1} \rightarrow 1, \quad k\left(x_{2}\right)\left(\frac{x_{2}}{h_{2}}\right)^{-1} \rightarrow 1 .
$$

Thus, terms in (2.4) go to 


$$
\left\{\begin{array}{ccc}
\left(\begin{array}{l}
l \\
p
\end{array}\right) x_{2}^{p}\left(x_{1} A\right)^{l-p} U_{0, l} & \text { if } & r=l-p, \\
0 & & r<l-p .
\end{array}\right.
$$

These convergence is uniform in $\left(x_{1}, x_{2}\right)$ on any bounded set of $R^{2}$. This shows the proposition.

Proof of Theorem. From the assumption that $U_{0}\left(x_{2}\right)$ is analytic at $x_{2}=0$, then we can set

$$
U_{0}\left(x_{2}\right)=\sum_{l=0}^{\infty} x_{2}^{l} U_{0, l},
$$

where $U_{0, l}$ are constant $m$-vectors. The assumption that the radius of convergence is $r_{0}$ leads us to the following estimate:

$$
\left\|U_{0, l}\right\| \leqq M r_{0}^{-l} \text {. }
$$

From (2.2) we have

$$
\begin{aligned}
& \hat{U}_{j\left(x_{1}\right), k\left(x_{2}\right)} \\
& =\sum_{q=0}^{\jmath\left(x_{1}\right)}\left(\begin{array}{c}
j\left(x_{1}\right) \\
q
\end{array}\right)(1-\tau A)^{\jmath\left(x_{1}\right)-q}(\tau A)^{q} \sum_{l=0}^{\infty}\left(k\left(x_{2}\right)+q\right)^{l} h_{2}^{l} U_{0, l} \\
& =\sum_{l=0}^{\infty} \sum_{q=0}^{j\left(x_{1}\right)}\left(\begin{array}{c}
j\left(x_{1}\right) \\
q
\end{array}\right)(1-\tau A)^{j\left(x_{1}\right)-q}(\tau A)^{q}\left(k\left(x_{2}\right)+q\right)^{l} h_{2}^{l} U_{0, l} \\
& =\sum_{l=0}^{\infty} \sum_{p=0}^{l} \sum_{r=1}^{\operatorname{Min}\left(j\left(x_{1}\right), l-p\right)}\left(\begin{array}{l}
l \\
p
\end{array}\right) j\left(x_{1}\right)\left(j\left(x_{1}\right)-1\right) \cdots\left(j\left(x_{1}\right)-r+1\right) C_{l-p, r} k\left(x_{2}\right)^{p} h_{2}^{l}(\tau A)^{r} U_{0, l} .
\end{aligned}
$$

For convenience sake we denote each term by $V_{l, p, r, x_{1}, x_{2}}$. By (1.3), (1.4) we have

$$
\begin{aligned}
& \left|\left(j\left(x_{1}\right)-q+1\right)\left(\frac{x_{1}}{h_{1}}\right)^{-1}\right| \leqq 1 \quad 1 \leqq q \leqq r \leqq \operatorname{Min}\left(j\left(x_{1}\right), l-p\right), \\
& \left|k\left(x_{2}\right)\left(\frac{x_{2}}{h_{2}}\right)^{-1}\right| \leqq 1
\end{aligned}
$$

Also, by (2.4) and (2.7), we have

$$
\left\|V_{l, p, r, x_{1}, x_{2}}\right\| \leqq\left(\begin{array}{l}
l \\
p
\end{array}\right) r^{l-p-r}\left(\begin{array}{l}
l-p-1 \\
r-1
\end{array}\right)\left|h_{2}\right|^{l-p-r}\left|x_{2}\right|^{p}\left(x_{1}\|A\|\right)^{r} M r_{0}^{-l} .
$$

For $1 \leqq r \leqq \operatorname{Min}\left(j\left(x_{1}\right), l-p\right)$

$$
\begin{aligned}
r^{l-p-r}\left|h_{2}\right|^{l-p-r} & \leqq j\left(x_{1}\right)^{l-p-r}\left|\frac{x_{1}}{h_{1}}\right|^{-(l-p-r)} x_{1}^{l-p-r}\left|\frac{h_{2}}{h_{1}}\right|^{l-p-r} \\
& \leqq x_{1}^{l-p-r}|\tau|^{-l+p-r}
\end{aligned}
$$

Thus we have 


$$
\begin{aligned}
& \left\|V_{l, p, r, x_{1}, x_{2}}\right\| \leqq\left(\begin{array}{c}
l \\
p
\end{array}\right)\left(\begin{array}{c}
l-p-1 \\
r-1
\end{array}\right)\left(\frac{x_{1}}{|\tau|}\right)^{l-p-r}\left(x_{1}\|A\|\right)^{r}\left|x_{2}\right|^{p} M r_{0}^{-l}, \\
& \|_{\sum_{r=1}^{\operatorname{Min}\left(j\left(x_{1}\right), l-p\right)} V_{l, p, r, x_{1}, x_{2}} \| \leqq\left(\begin{array}{c}
l \\
p
\end{array}\right)\left\{\frac{x_{1}}{|\tau|}+x_{1}\|A\|\right\}^{l-p}\left|x_{2}\right|^{p} M r_{0}^{-l} .}
\end{aligned}
$$

So, we have

$$
\left\|\sum_{p=0}^{l} \sum_{r=1}^{\operatorname{Min}\left(\jmath\left(x_{1}\right), l-p\right)} V_{l, p, r, x_{1}, x_{2}}\right\| \leqq M\left\{\left|x_{2}\right|+\frac{1+|\tau|\|A\|}{|\tau|} x_{1}\right\}^{l} r_{0}^{-l} .
$$

If $\left(x_{1}, x_{2}\right)$ is in $D_{\tau, r_{1}}$ then we have

$$
\left\|\sum_{p=0}^{l} \sum_{r=1}^{\operatorname{Min}\left(j\left(x_{1}\right), l-p\right)} V_{l, p, r, x_{1}, x_{2}}\right\| \leqq M\left(\frac{r_{1}}{r_{0}}\right)^{l} .
$$

The last estimate is independent of $N$ and is uniform in $\left(x_{1}, x_{2}\right)$ on $D_{\tau, r_{1}}$ By Proposition, we have

$$
\sum_{p=0}^{l} \sum_{r=1}^{\operatorname{Min}\left(j\left(x_{1}\right), l-p\right)} V_{l, p, r, x_{1}, x_{2}} \longrightarrow\left(x_{2}+x_{1} A\right)^{l} U_{0, l} .
$$

Summing up of all previous arguments, we conclude that $\hat{U}_{j\left(x_{1}\right), k\left(x_{2}\right)}$ goes to $U\left(x_{1}, x_{2}\right)=\sum_{l=0}^{\infty}\left(x_{2}+x_{1} A\right)^{l} U_{0, l}$ as $N \rightarrow \infty$. Moreover, this convergence is uniform on $D_{\tau, r_{1}}$.

\section{References}

[1] Courant, F., Friedrichs, K.O. and Lewy, H., Math. Über die partiellen Differenzengleichungen der mathematischen Physik, Math. Ann., 32 (1928), 100.

[2] Dahlquist, G., Convergence and stability for a hyperbolic difference equation with analytic initial values, Math, Scand. 2, (1954), 91-102. 